\title{
Ethnicity as predictor of immune reconstitution among Malaysian HIV-positive patients treated with highly active antiretroviral therapy
}

\begin{abstract}
The impact of sociodemographic and clinical factors on immune recovery and viral load suppression among HIV-1 positive patients treated with HAART particularly in Malaysia is largely unknown. This cross-sectional study enrolled 170 HIV-1-infected individuals of three major ethnicities who attended three HIV outpatient clinics in Malaysia. Questionnaire was used to obtain sociodemographic data while CD4 count and viral load data were gathered from hospital's record. Multiple factors were assessed for their predictive effects on CD4 count recovery ( $\geq 500$ cells $/ \mathrm{mm} 3$ ) and viral load suppression ( $\leq 50$ copies $/ \mathrm{mL}$ ) using binary logistic regression. Most of the subjects were male (149/87.6\%), in the age group 30 to 39 years old (78/45.9\%) and got infected via homosexual contact $(82 / 48.2 \%)$. Indians were associated with 11 times higher chance for CD4 recovery as compared to Malays at 8 to 12 months of HAART (adjusted OR: 10.948, 95\% CI: $1.873,64.001, \mathrm{P}=.008)$. Viral load suppression was positively influenced by intravenous drug use (IVDU) status (adjusted OR: 35.224, 95\% CI: 1.234, 1000.489, P =.037) at 4 to 6 months of HAART. Higher pretreatment CD4 count was a positive predictor for both initial immunological and virological responses while higher pretreatment viral load was a negative predictor for virological suppression only. In conclusion, ethnicity plays a significant role in determining early immune reconstitution in Malaysia, besides pretreatment CD4 count. Further studies are needed to identify possible biological factors underlying this association.
\end{abstract}

Keyword: HAART; HIV; Malaysia; Ethnicity; Immune reconstitution; Viral loaders 